\section{Influence of Epiphyses on the Regulation of Bone Growth}

Growth in length of a long bone occurs at epiphysial cartilage plates. Where more than one epiphysis is concerned, does each make its contribution irrespective of the other or does the growth of one influence that of the other?

During an investigation of the effect of irradiation on epiphysial growth, Reidy found that there was a stimulation of growth at the untreated epiphysis of a bone after growth at the other end had been impaired ${ }^{1}$. This suggested that there is some link between epiphyses, and prompted a series of experiments with tibiae of rabbits. Initially the proximal epiphysial plate of one tibia was destroyed using a dental drill, and subsequent growth of each epiphysis was followed to maturity by radiography, measurements being made from an opaque marker placed in the diaphysis's.

Where cessation of growth at the proximal epiphysis was achieved, the distal epiphysis made a contribution to length up to 27 per cent greater than that of the control tibia. A similar but less marked response was seen at the proximal tibial epiphysis when the distal epiphysis of the fused tibia and fibula was destroyed.

Destruction of an epiphysis by this method leads to the formation of a bony bridge between epiphysis and diaphysis, which must be assumed to provide both a means of communication not present in the control tibia and an alteration in the vascular supply of the epiphysis and diaphysis. It was also not known for certain whether the change in growth rate after destruction of one epiphysis was limited to the experimental bone or whether a similar change might be found elsewhere in the limb. With these possibilities in mind, further experiments have been carried out.

To diminish the factor of trauma at the epiphysis to be arrested, growth was limited mechanically, using a pair of stainless steel staples placed medially and laterally across the upper tibial epiphysial cartilage plate, thus linking epiphysial to metaphysial bone. In a further group of animals which had undergone this treatment, growth of the lower femoral epiphysis was also followed from an additional marker placed in the shaft of the femur. Effective arrest of growth at the upper tibial epiphysis was found to be produced by the staples and an increase in contribution to growth in length similar to that found after destruction of the epiphysial plate was observed at the lower tibial epiphysis on the experimental side.

Histological preparations also showed that after stapling, the epiphysial plate, although narrowed, remained intact and that no abnormal vascular reaction was present around the portion of the staple embedded in cortical or trabecular bone.

Maturity was reached in seven of the animals in which a fornoral marker was used together with upper tibial epiphysial stapling. In five I recorded an increase of 15 per cent or more (maximum 26 per cent) of growth at the uninjured epiphysis on the experimental side, but in no animal was the lower fomoral epiphysis adjacent to the arrested tibial epiphysis found to contribute more to femoral growth than that on the contralateral side.

Throughout these experiments not all attempts to produce arrest of growth were uniformly successful and it soon became apparent that the degree of arrest of one epiphysis was related to the excess contribution made by the epiphysis at the other end of the bone. Fig. 1 shows that the percentage deficits produced at the proximal tibial epiphysis are proportional to the percentage increase made by the distal epiphysis on the experimental side.

Following up these findings the growth curves of those animals in which the staples had cut out were reviewed and, in a further group, staples were intentionally removed after the expected growth changes at the distal experimental epiphysis had become manifest. After release from the staples growth was resumed at the previously arrested upper epiphysis and at the lower epiphysis at rates nearly comparable with those of the contralateral epiphyses. The growth rate of the released epiphysis, however, was not found to exceed that of its contralateral control. This finding might well mean that, as in the normal bone, there is a limit for the combined rates of growth of the two epiphyses. It has been shown that both may increase their rate when they are the sole active site of growth, but this does not seem possible when both are active even though there is a defect in total bone length.

These experiments suggest that there may be a system of growth eontrol intrinsic to a bone. This system seems to respond to changes in growth rate of the individual epiphyses of that bone in a manner which tends to maintain the rate of growth of the bone as a whole.

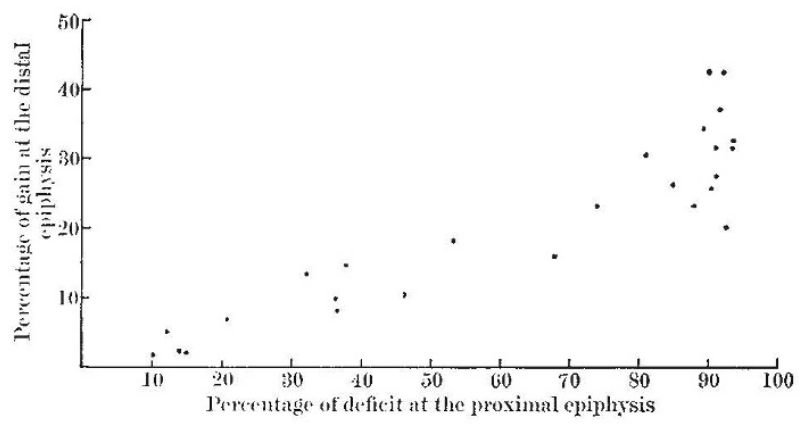

Fig. 1. Percentage gain at the distal tibial epiphysis plotted against percentage deficit at the proximal arrested epiphysis in twenty-six experimental animals.

The defect in growth at one epiphysis seems to produce a directly proportional additional growth at another epiphysis in the same bone, but it does not stimulate growth in the adjacent epiphysis of another bone. It is not yet possible to say how control in this system is mediated. It may be that there is a maximum potential for the growth of a bone which cannot be exceeded but may be possessed to a greater extent by one epiphysis when a second ceases to grow. This potential could be in the form of an essential metabolite of limited supply. On the other hand, an inhibitory agent may be released locally in the process of chondrocyte multiplication or ossification, with a concentration which decreases after the destruction or arrest of an epiphysis.

Department of Anatomy,

\section{E, C, B, HALL-CRAGGS}

University College London.

Received November 6, 1968; revised February 6, 1969.

'Reidy, J. A., Lingley, J. R., Gall, E. A., and Barr, J. S., J. Bone Joint Surg., 43B, 121 (1947)

${ }^{2}$ Hall-Craggs, E. C. B., J. Bone Joint Surg., 50B, 392 (1968).

\section{Free Radical Reactions modify Cellular Damage}

СомвINED physical and biological techniques reveal some properties of molecular species which are responsible for cell death after irradiation. Changes in ESR spectra of irradiated cell contents and in the survival of irradiated fern spores, produced by different gases at different times, show an important correlation. The primary radiationproduced radical population has been shown to include precursors of ultimate biological damage, which are still 